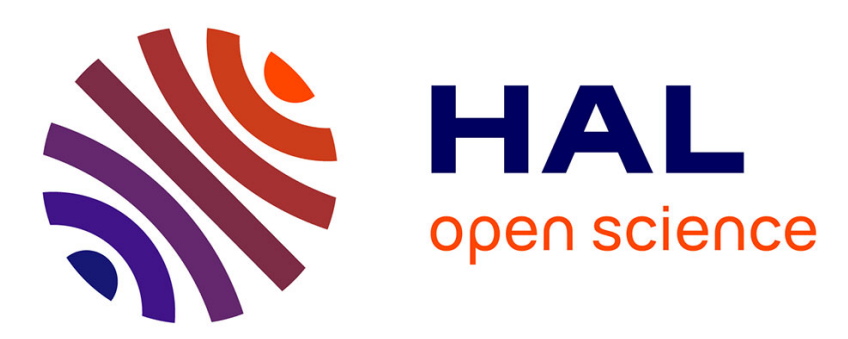

\title{
Quelques modèles de calcul de pertes supplémentaires dans les bobinages des transformateurs de puissance
}

T. Ngnegueu, G. Meunier, J.-C. Sabonnadière, M. Mailhot, J.-P. Arthaud

\section{To cite this version:}

T. Ngnegueu, G. Meunier, J.-C. Sabonnadière, M. Mailhot, J.-P. Arthaud. Quelques modèles de calcul de pertes supplémentaires dans les bobinages des transformateurs de puissance. Revue de Physique Appliquée, 1990, 25 (3), pp.295-311. 10.1051/rphysap:01990002503029500 . jpa-00246188

\section{HAL Id: jpa-00246188 https://hal.science/jpa-00246188}

Submitted on 1 Jan 1990

HAL is a multi-disciplinary open access archive for the deposit and dissemination of scientific research documents, whether they are published or not. The documents may come from teaching and research institutions in France or abroad, or from public or private research centers.
L'archive ouverte pluridisciplinaire HAL, est destinée au dépôt et à la diffusion de documents scientifiques de niveau recherche, publiés ou non, émanant des établissements d'enseignement et de recherche français ou étrangers, des laboratoires publics ou privés. 


\title{
Quelques modèles de calcul de pertes supplémentaires dans les bobinages des transformateurs de puissance
}

\author{
T. Ngnegueu ( $\left.{ }^{1}\right)$, G. Meunier ( $\left.{ }^{1}\right)$, J.-C. Sabonnadière $\left({ }^{1}\right)$, M. Mailhot $\left({ }^{2}\right)$ et J.-P. Arthaud ( $\left.{ }^{2}\right)$ \\ (1) Laboratoire d'Electrotechnique de Grenoble (UA CNRS 355), E.N.S.I.E.G. Domaine Universitaire, \\ BP 46, 38402 St Martin d'Hères, France \\ (2) Jeumont-Schneider, Division Transformateurs de Puissance, 84 Avenue Paul Santy, 69371 Lyon Cedex 08, \\ France
}

(Reçu le 6 avril 1989, révisé le 24 août 1989 et le 23 novembre 1989, accepté le 24 novembre 1989)

\begin{abstract}
Résumé. - Nous présentons quelques modèles de calcul de pertes supplémentaires dans les bobinages des transformateurs de puissance. Nous analysons les pertes par courants de Foucault et les pertes par courants de circulation, tout en prenant les transpositions en compte. Enfin, aux différentes étapes, nous présentons des comparaisons entre des résultats de calcul et des résultats d'essais.
\end{abstract}

\begin{abstract}
In this paper, we present several methods, for the calculation of extra losses in power transformers' windings. Eddy current losses and circulating current losses are investigated. Transpositions are also taken into account. At various steps, we also present some comparisons between our calculations and test results.
\end{abstract}

\section{Introduction.}

Le présent article résume une étude menée conjointement par le Laboratoire d'Electrotechnique de Grenoble et la division transformateurs de puissance de la compagnie Jeumont-Schneider à Lyon. Son but est le calcul des pertes supplémentaires dans les transformateurs de puissance. Ces pertes sont localisées dans le circuit magnétique, dans la cuve et dans le bobinage. $\mathrm{Ce}$ sont ces dernières qui seront analysées dans la suite.

Une attention particulière est portée au cas des transformateurs de type cuirassé qui sont des transformateurs dans lesquels, le circuit magnétique

est d'assurer un bon serrage mécanique des bobines. Pour une bonne représentation des pertes supplémentaires, une description précise du transformateur est nécessaire. La difficulté principale provient de la complexité de la géométrie, liée au nombre de brins conducteurs qui constituent une phase.

Les bobines d'une phase sont constituées de plusieurs spires qui peuvent elles-mêmes être constituées de plusieurs couches de conducteurs subdivisées en brins électriquement isolés. Le nombre de couches de conducteurs est d'autant plus grand en basse tension que le rapport de transformation du transformateur est élevé. Dans le logiciel que nous avons développé à l'occasion de cette étude, nous avons essayé de donner la meilleure description possible des différentes fenêtres du transformateur, afin de pouvoir identifier et suivre chaque brin conducteur dans une phase du transformateur.

\section{Calcul des pertes dans les bobines.}

Les pertes dans le bobinage peuvent être décomposées en pertes par effet Joule qui sont dues au passage du courant de charge, et en pertes supplémentaires qui sont dues au passage des courants $\Sigma_{\text {brins }} R_{\text {brin }}: I_{0_{\text {cff brin }}}^{2}$ où, $I_{0_{\text {cff brin }}}$ est le courant de charge efficace dans un brin conducteur. En pratique, les pertes par effet Joule sont obtenues par la mesure des résistances des bobines.

Les pertes supplémentaires dans les bobines, se décomposent en pertes dues aux courants de Foucault et en pertes dues aux courants de circulation lorsque les conducteurs sont subdivisés en brins court-circuités en parallèle. Dans les paragraphes ultérieurs, nous aurons l'occasion d'expliciter davantage la nature de chacune de ces pertes. 


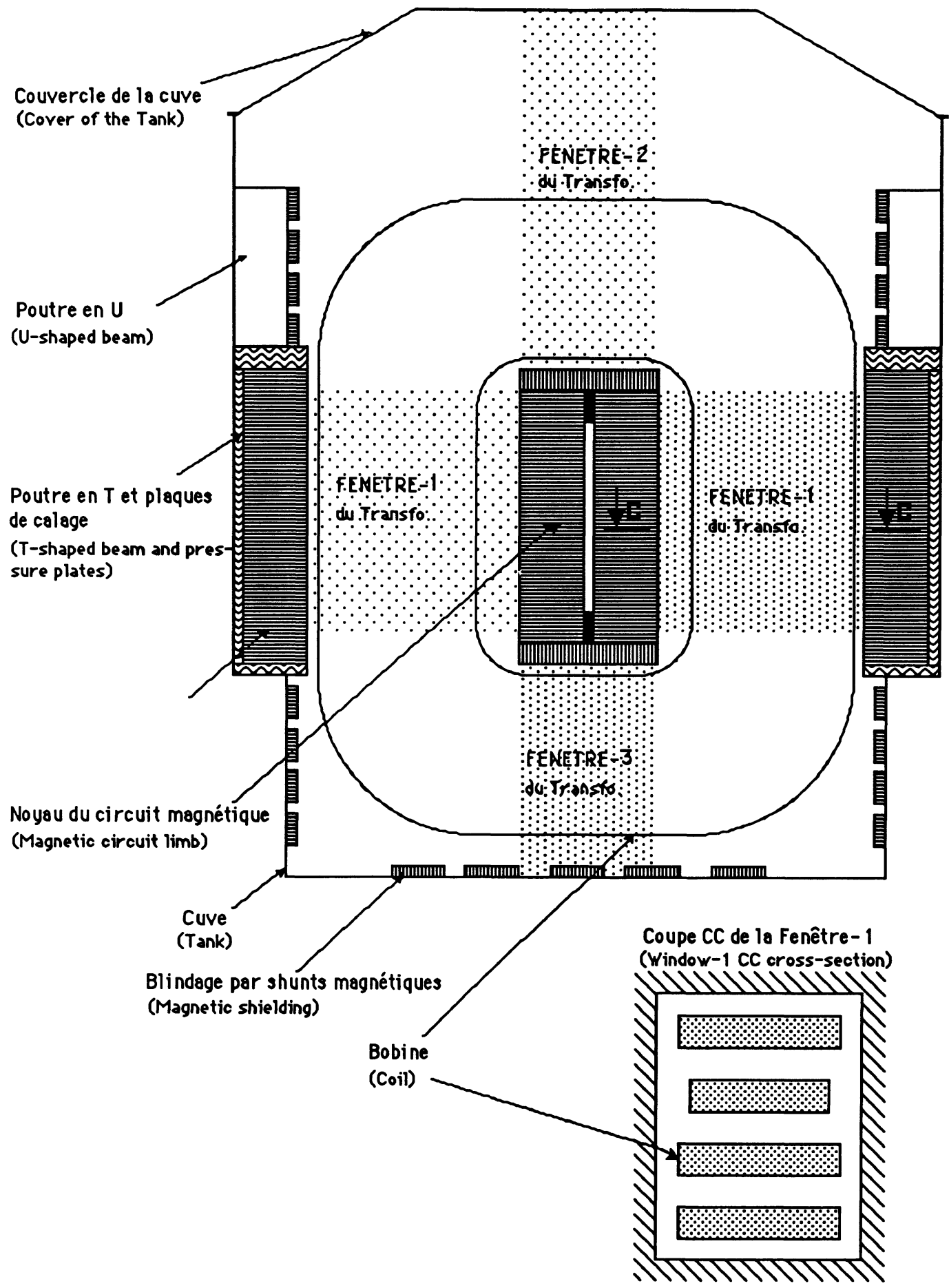

a)

Fig. 1. - Description schématique d'un transformateur monophasé de type cuirassé. (a) Quelques vues en coupe schématique d'un transformateur monophasé de type cuirassé. (b) Vue d'une bobine. (c) Coupe d'une spire avec deux couches de conducteurs en parallèle. (d) Coupe d'un conducteur subdivisé en quatre brins.

[Diagrammatic description of a one phase shell form transformer. (a) Some diagrammatic sectional views of a one phase shell form transformer. (b) View of a coil. (c) Cross-section of a turn with two layers of conductors. (d) Cross-section of a four-strip conductor.] 


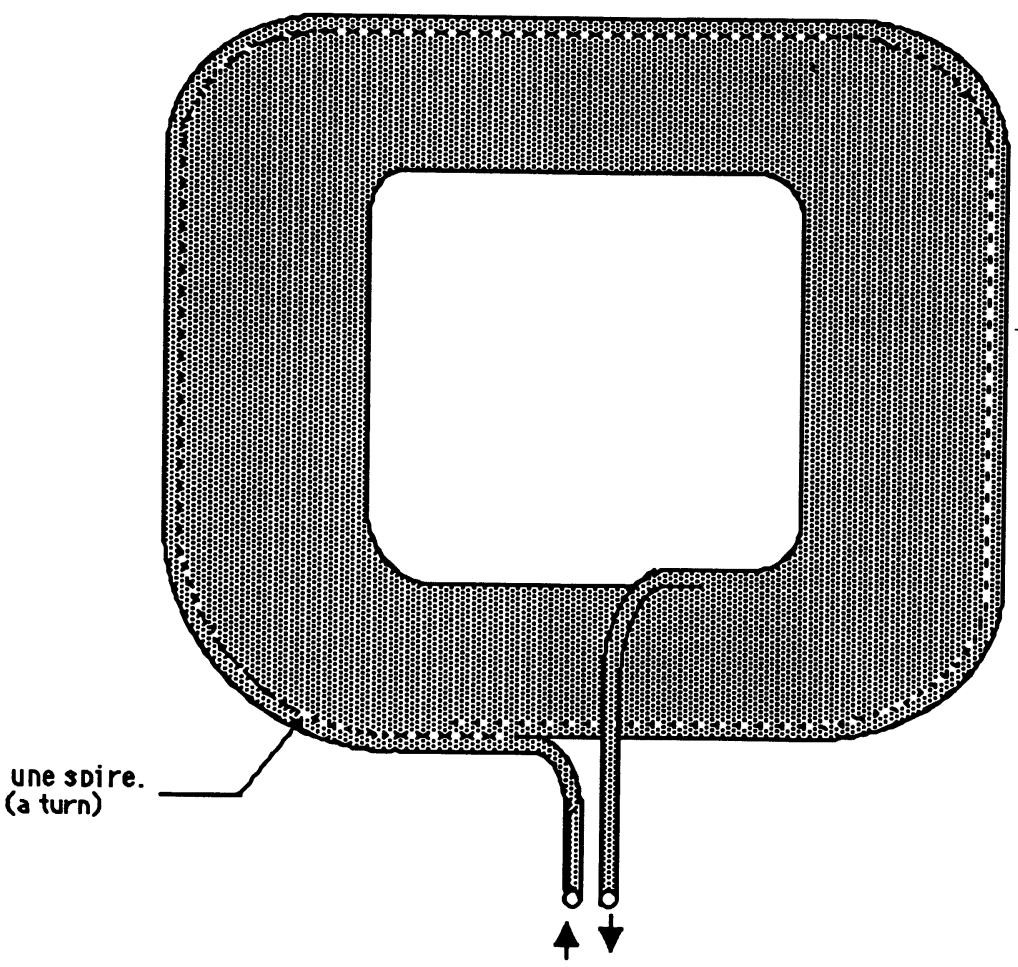

b)

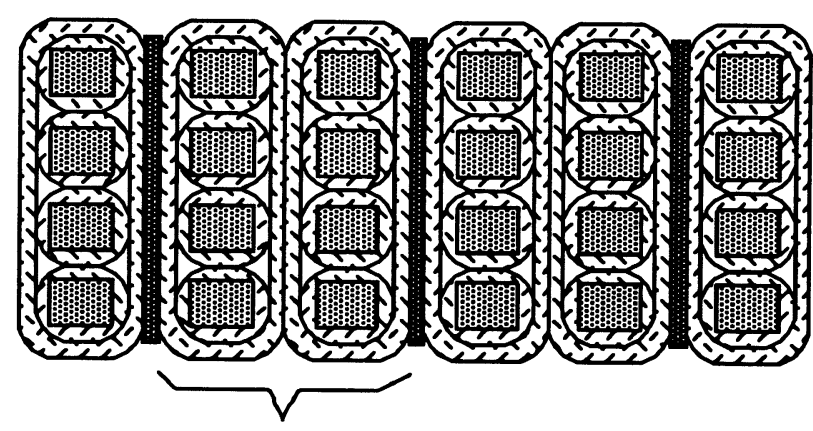

c)

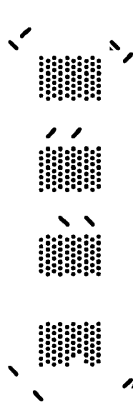

couche exterieure de papier enduit de produit thermo-durcissable.

(external paper layer with thermo-hardening product)

isolation commune aux quatre brins.

(common insulation of the four strips)

isolation individuelle d'un brin.

(individual insulation of a strip)

le brin conducteur élémentaire

(the elementary conducting strip)

d)

Fig. 1 (suite). 
Pour le calcul des pertes supplémentaires, il est possible d'utiliser des méthodes numériques comme par exemple la méthode des éléments finis en régime dynamique et ceci, d'autant que notre environnement de travail est propice à l'usage d'une telle méthode. Cependant, compte tenu du nombre de brins conducteurs dans une phase de transformateur de puissance et compte tenu des dimensions réduites de ces brins, une méthode de calcul comme la méthode des éléments finis exigerait un maillage très fin et conduirait à la résolution d'un système linéaire d'ordre très élevé. Par exemple, pour un gros transformateur commercial, on peut facilement atteindre 8000 brins conducteurs dans la coupe transversale d'une phase. Un" maillage en éléments finis du premier ordre, avec des mailles triangulaires conduirait déjà à 32000 nœuds pour ces seuls brins. Il faudrait ensuite procéder au maillage du reste de la structure. A cause du faible espacement des bobines, ce maillage devrait être assez fin si l'on veut obtenir des triangles de bonne qualité. En outre, pour avoir une bonne précision des résultats, des éléments finis d'ordre deux devraient être nécessaires, ce qui augmenterait encore le nombre de nœuds. Ces considérations donnent une idée de la taille que le problème pourrait atteindre. Par souci d'économiser du temps de calcul CPU, pour une meilleure précision des résultats et une certaine souplesse de travail, nous avons choisi une méthode de calcul analytique. Cette approche est possible grâce à l'absence de non-linéarités et à la simplicité des formes géométriques qui facilitent l'expression des conditions aux limites. La méthode analytique choisie s'appuie sur le calcul du champ de fuite dans la coupe des différentes fenêtres du transformateur en régime statique. Le principe de la méthode consiste à découpler l'évaluation du champ de fuite qui est la cause des courants induits, et l'évaluation des courants induits eux-mêmes. Une telle approche est possible parce que nous faisons l'hypothèse, sous réserve de vérifications, que le champ de réaction des courants induits est négligeable devant le champ de fuite inducteur. Les calculs peuvent être conduits en régime statique parce que nous supposons qu'il n'y a pas de pièces conductrices massives dans les différentes fenêtres du transformateur; de ce fait, dans une phase du transformateur, les différentes composantes du champ de fuite en un point tournent en phase ou en opposition de phase.

2.1 Modèle DE CAlCul anAlytiQue du Champ DE FUITE DANS LA COUPE D'UNE FENÊTRE DE TRANSFORMATEUR DE PUISSANCE DE TYPE CUIRASSÉ, EN RÉGIME STATIQUE. - Les dimensions et la forme particulière de ce type de transformateur de puissance permettent une approche bidimensionnelle du problème. Le transformateur de puissance est alors considéré comme un ensemble de quatre fenêtres rectangulaires dont deux sont identiques (Fig. 1a). Les équations du champ de fuite dans une fenêtre du transformateur sont obtenues à partir des équations de Maxwell pour les régimes quasi-stationnaires :

$$
\begin{aligned}
\text { Rot } \mathbf{H} & =\mathbf{J} \\
\text { Div } \mathbf{B} & =0 \\
\text { B } & =\mu \mathbf{H}
\end{aligned}
$$

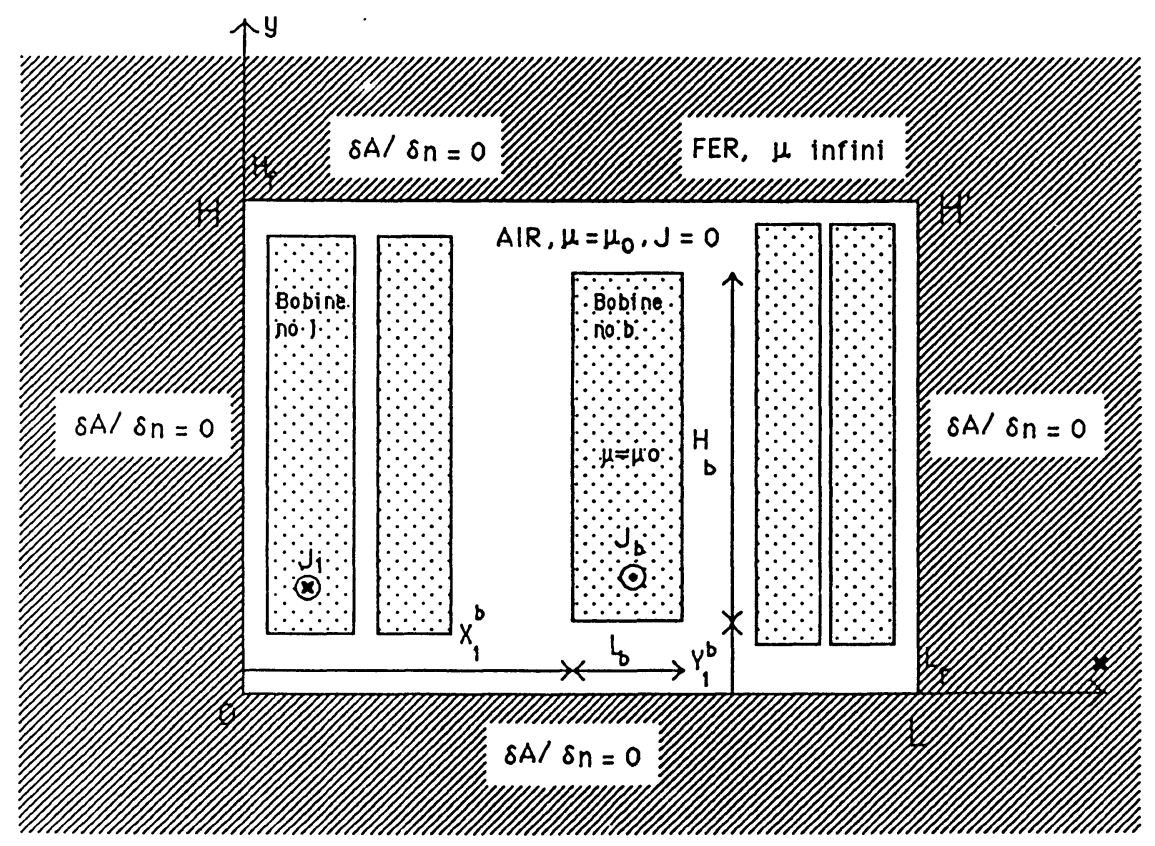

Fig. 2. - Vue paramétrée de la coupe d'une fenêtre en vue du calcul du champ de fuite.

[Sectional view of a window for the calculation of the leakage flux.] 
Dans ces équations, $\mathbf{H}$ est le champ magnétique, $\mathbf{J}$ est la densité de courant, B est l'induction magnétique et $\mu$ est la perméabilité magnétique, ici supposée constante et égale à $\mu_{0}$, la perméabilité magnétique de l'air.

La relation (2) permet de définir un potentiel vecteur A par la relation,

$$
\operatorname{Rot} \mathbf{A}=\mathbf{B}
$$

à laquelle nous adjoignons la condition de jauge,

$$
\operatorname{Div} \mathbf{A}=0
$$

A partir des équations (1), (3), (4) et (5), nous obtenons l'équation régissant la répartition du potentiel vecteur A par:

$$
\Delta \mathbf{A}=-\mu_{0} \mathbf{J}
$$

En régime statique, la densité de courant de charge $\mathbf{J}$ est orientée suivant l'axe $(\mathrm{O} z)$. L'induction magnétique B étant dans le plan $(\mathrm{O} x, \mathrm{O} y)$, le potentiel vecteur A est porté par l'axe $(\mathrm{Oz})$. L'équation (6) devient alors une équation scalaire :

$$
\Delta A=-\mu_{0} J
$$

et la condition de jauge (5) qui se réduit à $\partial A / \partial z=0$, se trouve automatiquement vérifiée.

Si nous négligeons les courants magnétisants, on peut considérer que la somme des courants dans chaque fenêtre du transformateur est nulle. De ce fait, pour une frontière rectangulaire et pour une perméabilité magnétique infinie pour le fer, on peut imposer les conditions aux limites suivantes :

$\partial A / \partial x=0$ sur la portion de frontière formée par le segment $[O, H]$ et par le segment $\left[L, H^{\prime}\right]$, et $\partial A / \partial y=0$ sur la portion de frontière formée par le segment $[O, L]$ et par le segment $\left[H, H^{\prime}\right]$.

Il est alors facile de vérifier que ces conditions de champ normal à l'interface entre la région FER et la région AIR sont compatibles avec la relation d'Ampère.

Après un développement de l'équation (7) en série double de Fourier et compte tenu des condipar l'expression :

$$
A(x, y)=\sum_{i=1}^{\infty} \sum_{j=1}^{\infty} A_{i j} \cos \left(m_{i} \cdot x\right) \cos \left(n_{j} \cdot y\right)
$$

où le coefficient $A_{11}$ peut être pris égal à zéro puisque le potentiel $A$ est défini à une constante près. Les autres coefficients $A_{m n}, n_{x}$ et $n_{y}$ sont obtenus en fonction des courants de charge et des paramètres géométriques de la fenêtre par les expressions suivantes :
Pour $i=1$, ou pour $j=1$,

$$
\begin{aligned}
A_{i 1} & =\frac{4}{\left(H_{\mathrm{f}} L_{\mathrm{f}} m_{i}^{3}\right)} \times \\
& \times \sum_{b=1}^{N_{b}} J_{b} L_{b} \sin \left(\frac{m_{i} H_{b}}{2}\right) \cos \left(m_{i}\left(\frac{H_{b}}{2}+Y_{1}^{b}\right)\right) \\
A_{1 j} & =\frac{4}{\left(H_{\mathrm{f}} L_{\mathrm{f}} m_{j}^{3}\right)} \times \\
& \times \sum_{b=1}^{N_{b}} J_{b} H_{b} \sin \left(\frac{n_{j} L_{b}}{2}\right) \cos \left(n_{j}\left(\frac{L_{b}}{2}+X_{1}^{b}\right)\right)
\end{aligned}
$$

et pour $i$ et $j$ différents de 1 ,

$$
\begin{aligned}
A_{i j}= & \frac{16}{H_{\mathrm{f}} L_{\mathrm{f} m_{i} n_{j}}\left(m_{i}^{2}+n_{j}^{2}\right)} \\
& \times \sum_{b=1}^{N_{b}} J_{b} \sin \left(\frac{m_{i} H_{b}}{2}\right) \cos \left(m_{i}\left(\frac{H_{b}}{2}+Y_{1}^{b}\right)\right) \\
& \times \sin \left(\frac{n_{j} L_{b}}{2}\right) \cos \left(n_{j}\left(\frac{L_{b}}{2}+X_{1}^{b}\right)\right) .
\end{aligned}
$$

Dans ces expressions, $m_{i}$ et $n_{j}$ sont obtenus après l'expression des conditions aux limites par: $m_{i}=$ $(i-1) \pi / H_{\mathrm{f}}$, et $n_{j}=(j-1) \pi / L_{\mathrm{f}}$.

D'autre part, $N_{b}$ représente le nombre total de bobines dans la fenêtre et $J_{b}$ la valeur algébrique de la densité moyenne de courant dans la bobine numéro $b$. Les autres paramètres géométriques $L_{\mathrm{f}}, H_{\mathrm{f}}, L_{b}, H_{b}, Y_{1}^{b}$ et $X_{1}^{b}$ sont définis sur la figure 3.

Pour le calcul de $A(x, y)$, en théorie, la sommation sur $i$ et $j$ doit se faire de 1 à l'infini, mais comme le montrent les expressions des coefficients $A_{i j}$, la série double donnée par l'expression (8) converge assez rapidement et il suffit de faire varier $i$ et $j$ de 1 à 15 .

Des expressions (8) et (4), on déduit le champ de fuite dans les parties rectilignes des différentes fenêtres du transformateur. Dans les portions arrondies des bobines situées entre deux fenêtres consécutives, le champ de fuite est obtenu par interpolation. De cette manière le champ de fuite est obtenu en tout point du bobinage. Nous avons fait des calculs en considérant $J_{b}$ comme la densité moyenne de courant dans les bobines. Nous avons ensuite considéré la répartition réelle de la densité de courant

coup plus long dans le second cas où on est amené à considérer individuellement chaque brin conducteur. Néanmoins, pour les maquettes de transformateurs qui nous ont servi de support, la différence des valeurs du champ de fuite n'est pas notable. Dans nos calculs, nous avons alors considéré que chaque bobine est parcourue par un courant dont la densité $J_{b}$ est égale à la densité moyenne de courant dans cette bobine. Cependant, la possibilité de pouvoir prendre en compte le courant de chaque brin conducteur élémentaire nous semble très intéres- 

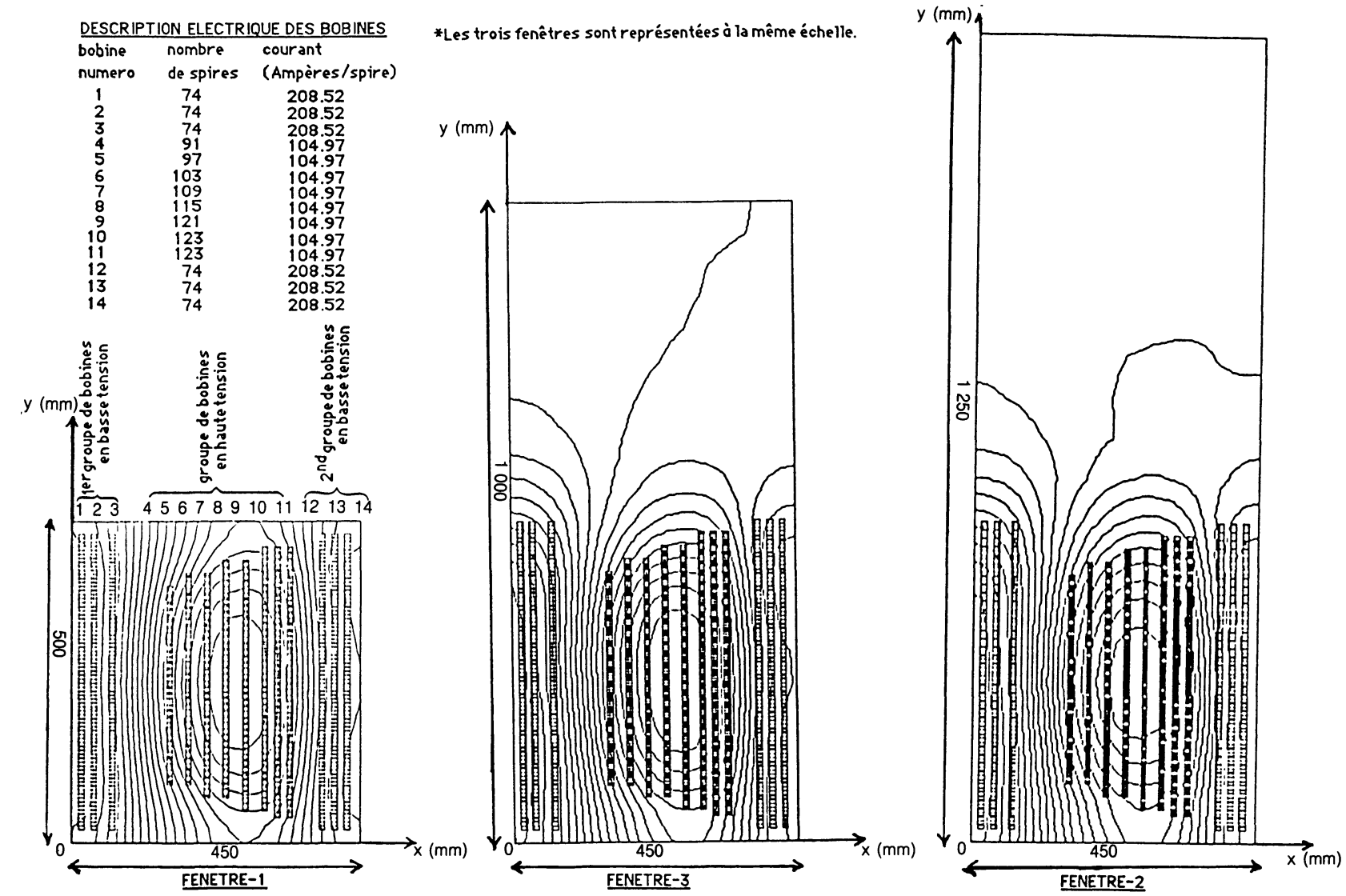

Fig. 3. - Lignes de champ dans les fenêtres d'un transformateur cuirassé de 13.333 MVA, d'après la méthode de calcul analytique.

[Lines of flux plotting in the windows of a 13.333 MVA shell form transformer following the analytical calculation.]

sante; nous avons essayé d'en tirer parti dans l'analyse des courants de circulation.

Le champ de fuite en régime statique tel qu'il vient d'être calculé peut également être obtenu par la méthode des éléments finis. Ce calcul a été fait en utilisant le logiciel FLUXLAB-2D qui est développé au Laboratoire d'Electrotechnique de Grenoble. Les valeurs du champ magnétique de fuite obtenues par la méthode des éléments finis sont comparables à celles obtenues par la méthode de calcul analytique qui vient d'être exposée.

Nous avons néanmoins noté en comparant les valeurs du champ de fuite issues des deux méthodes de calcul que, la précision des calculs, médiocre pour les points où, le module du champ est presque nul, est de l'ordre de quelques pour cent partout ailleurs.

\subsection{MOdÈle DE CAlCul DES PERTES PAR COU- RANTS DE FOUCAULT.}

2.2.1 Analyse du brin élémentaire. - Par courants de Foucault, nous voulons désigner les courants induits dont le trajet se referme à l'intérieur d'un même brin conducteur. Pour l'analyse des pertes par courants de Foucault, un brin conducteur unique, de section rectangulaire et de longueur infinie est examiné. Ce brin conducteur est placé sous l'influence d'un champ de fuite $\mathbf{H}_{0}$. Les dimensions du brin conducteur étant très petites devant les dimensions du transformateur, on peut les considérer comme étant de l'ordre de la dimension élémentaire dans ce qui pourrait être un processus de calcul numérique. De la sorte, le champ de fuite peut être considéré comme uniforme à l'échelle du conducteur élémentaire. Dans l'approximation bidimensionnelle, ce champ de fuite se trouve dans le plan de la section du conducteur; nous désignons $(\mathrm{O} x, \mathrm{O} y)$ un tel plan. Les dimensions du brin conducteur étant

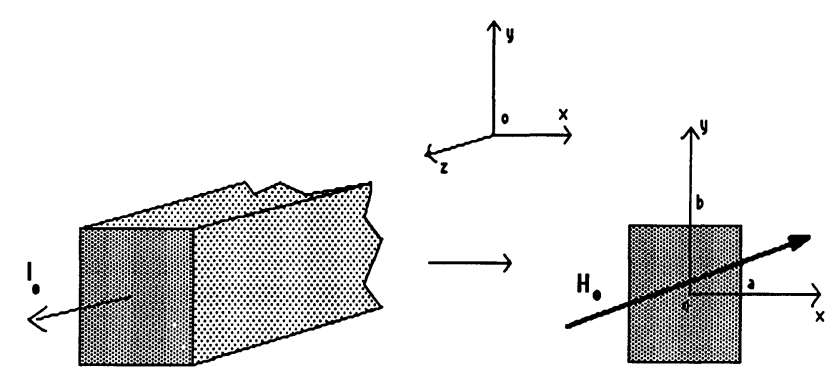

Fig. 4. - Le brin conducteur élémentaire dans le champ de fuite.

[The individual conducting strip in the leakage flux.] 
supposées inférieures à l'épaisseur de peau, nous faisons l'hypothèse que le champ de réaction des courants de Foucault est négligeable devant le champ inducteur $\mathbf{H}_{0}$; ce qui nous permet de considérer que le champ vaut, en direction et en module, $\mathbf{H}_{0}$ tout autour du conducteur élémentaire. Une fois la répartition des courants de Foucault calculée, nous vérifions la validité de cette hypothèse.

A partir des équations de Maxwell et des conditions aux limites que nous venons de définir, on trouve une répartition de la densité des courants de Foucault portée par l'axe $(\mathrm{Oz})$ et donnée en amplitude complexe par l'expression ! :

$$
\begin{aligned}
J_{\mathrm{f}}(x, y)=\frac{(i+1) H_{0 y}}{\delta} & \frac{\operatorname{sh}\left(\frac{i+1}{\delta} x\right)}{\operatorname{ch}\left(\frac{(i+1)}{\delta} a\right)}+ \\
& +\frac{(i+1) H_{0 x}}{\delta} \frac{\operatorname{sh}\left(\frac{i+1}{\delta} y\right)}{\operatorname{ch}\left(\frac{(i+1)}{\delta} b\right)}
\end{aligned}
$$

où $H_{0 x}$ ou $H_{0 y}$ est pris comme origine des phases. On constate que $J_{f}$, la densité de courants de Foucault a une intégrale nulle sur la section du conducteur. On montre alors aisément que les pertes dues aux courants de Foucault peuvent être calculées séparément des pertes par effet Joule. En effet, si un courant de densité $J_{0}$ uniforme sur la section du conducteur se superpose aux courants de Foucault, les pertes totales dans le conducteur sont données d'après le théorème de Poynting par la relation :

$$
W=\frac{1}{2 \sigma} \int_{S}\left|J_{0}+J_{\mathrm{f}}\right|^{2} \mathrm{~d} S
$$

soit en développant,

$$
\begin{aligned}
W=\frac{1}{2 \sigma}\left(\int_{S}\left|J_{0}\right|^{2} \mathrm{~d} S\right. & +J_{0}^{*} \int_{S} J_{\mathrm{f}} \mathrm{d} S+ \\
& \left.+J_{0} \int_{S} J_{\mathrm{f}}^{*} \mathrm{~d} S+\int_{S}\left|J_{\mathrm{f}}\right|^{2} \mathrm{~d} S\right)
\end{aligned}
$$

d'où,

$$
W=\frac{1}{2 \sigma} \int_{S}\left|J_{0}\right|^{2} \mathrm{~d} S+\frac{1}{2 \sigma} \int_{S}\left|J_{\mathrm{f}}\right|^{2} \mathrm{~d} S .
$$

f ar son expression, es pe es par courants de Foucault par unité de longueur du conducteur élémentaire sont exprimées par :

$$
\begin{aligned}
P_{\mathrm{f}}= & \frac{1}{\sigma \delta}\left(2 a \cdot H_{0 y^{2}} \frac{\frac{\operatorname{sh}(2 b)}{\delta}-\sin \left(\frac{2 b}{\delta}\right)}{\operatorname{ch}\left(\frac{2 b}{\delta}\right)+\cos \left(\frac{2 b}{\delta}\right)}+\right. \\
& \left.+2 b \cdot H_{0 x^{2}} \frac{\operatorname{sh}\left(\frac{2 a}{\delta}\right)-\sin \left(\frac{2 a}{\delta}\right)}{\operatorname{ch}\left(\frac{2 a}{\delta}\right)+\cos \left(\frac{2 a}{\delta}\right)}\right)
\end{aligned}
$$

$4 a b$ représentant la section rectangulaire du conducteur, $\delta$ l'épaisseur de peau, $\sigma$ la conductivité électrique et $\mathbf{H}_{0}$ le champ magnétique extérieur.

En suivant tous les brins conducteurs du transformateur, les pertes par courant de Foucault peuvent ainsi être calculées.

Il est intéressant de noter qu'en présence de plaque dans la fenêtre, il serait plus difficile de trouver une solution analytique au champ de fuite, et de plus, les deux composantes du champ magnétique extérieur $\mathbf{H}_{0}$ ne seraient plus en phase. Nous avons vérifié par comparaison avec les résultats donnés par le logiciel de calcul par la méthode des éléments finis FLUXLAB-2D que dans ce cas, l'expression précédente reste valable pour les conducteurs fins, c'est-à-dire des conducteurs dont les dimensions sont inférieures à l'épaisseur de peau, à condition de remplacer dans l'expression (9), $H_{0 x^{2}}$ et $H_{0 y^{2}}$ qui deviennent des grandeurs complexes par leurs modules.

2.2.2 Analyse du champ de réaction des courants de Foucault. - Dans le paragraphe précédent, nous avons formulé l'hypothèse que, lorsque les dimensions du brin conducteur sont inférieures à l'épaisseur de peau, on peut négliger le champ de réaction des courants de Foucault devant le champ de fuite inducteur. Cette hypothèse nous a permis d'affecter la valeur $\mathbf{H}_{0}$ au champ autour du conducteur. Dans ce paragraphe, il s'agit de vérifier cette hypothèse. Sur la figure (5), nous effectuons une comparaison entre le champ $\mathbf{H}$ créé par les courants de Foucault et le champ appliqué $\mathbf{H}_{0}$. Pour ce calcul nous avons choisi de considérer un champ inducteur $\mathbf{H}_{0}$ tel que ses deux composantes $H_{0 x}$ et $H_{0 y}$ soient égales. De plus nous avons choisi un conducteur de section carré de telle sorte qu'il soit suffisant pour des raisons de symétrie évidentes, de prendre les points de calcul sur une seule de ses faces.

Ainsi, dans les figures qui suivent, après avoir appliqué un champ inducteur $\mathbf{H}_{0}$ au conducteur de section carré, nous calculons les courants induits dans ce conducteur élémentaire sans tenir compte du champ produit par les courants induits. Ensuite, nous nous plaçons sur une face du conducteur ; nous c oisissons par exemp e, une es aces parallèle au plan $(\mathrm{O} x, \mathrm{O} z)$. En différents points de ce plan, nous calculons les deux composantes du champ $\mathbf{H}$ produit par les courants de Foucault et les comparons au champ appliqué $\mathbf{H}_{0}$; on constate que le rapport du module du champ de réaction des courants induits à celui du champ appliqué est faible lorsque les dimensions de la section de conducteur sont de l'ordre l'épaisseur de peau et que ce rapport devient vite négligeable lorsque les dimensions de la section du conducteur deviennent de l'ordre de quelques fractions de l'épaisseur de peau. 
$\ldots$ — $|\mathrm{Hx}| /\left|\mathrm{Ho}_{\mathrm{O}}\right| \ldots . . . . . .:|\mathrm{Hy}| /|\mathrm{Ho}|$

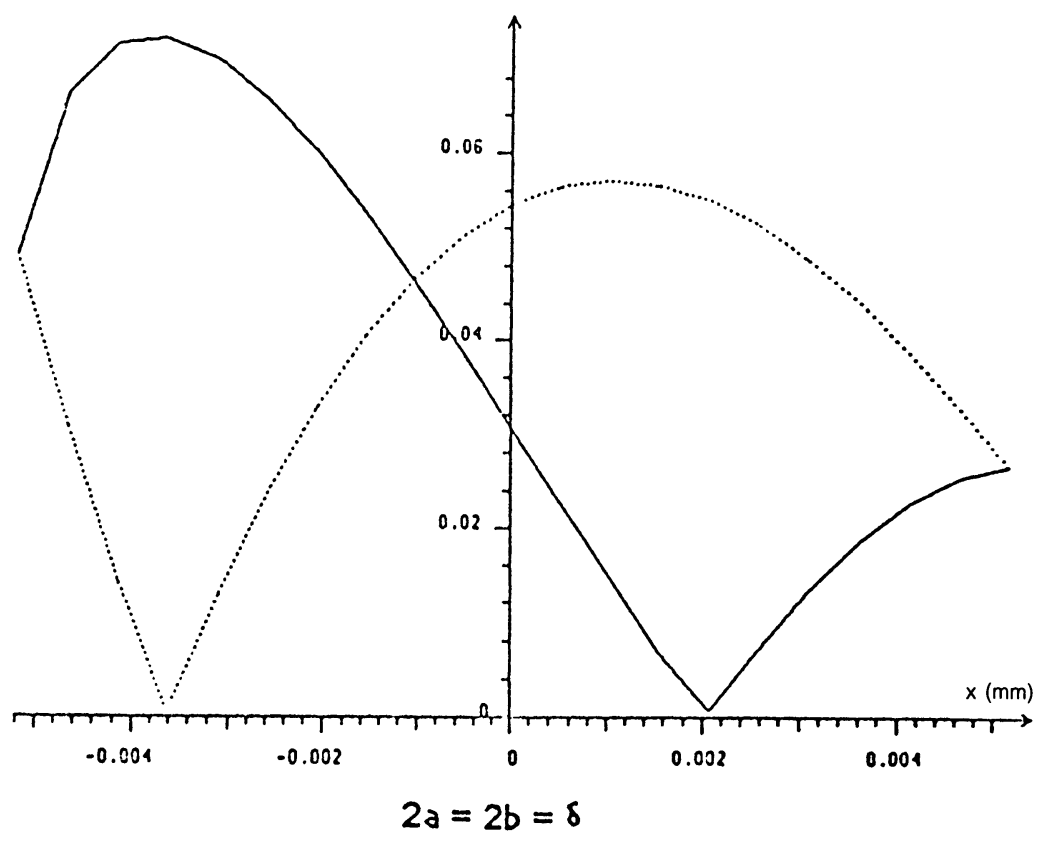

- $|\mathrm{Hx}| /|\mathrm{Ho}| \ldots \ldots \ldots .:|\mathrm{Hy}| /|\mathrm{Ho}|$

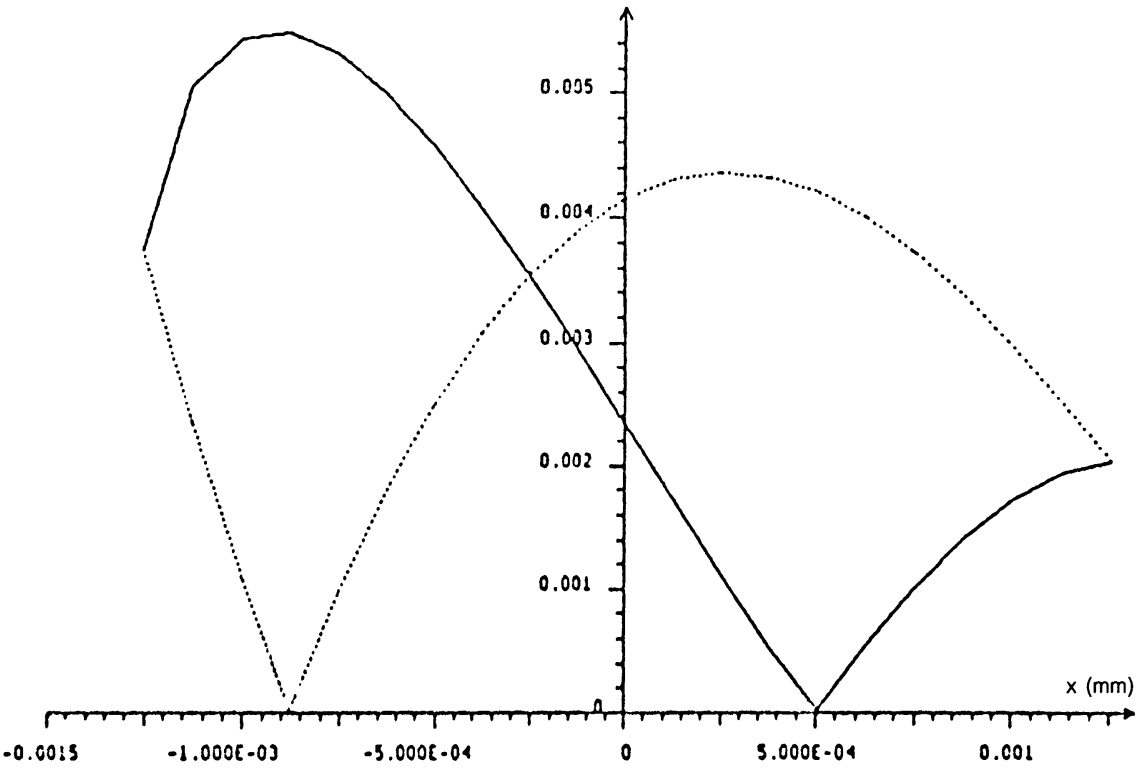

$2 a=2 b=\delta / 4$.

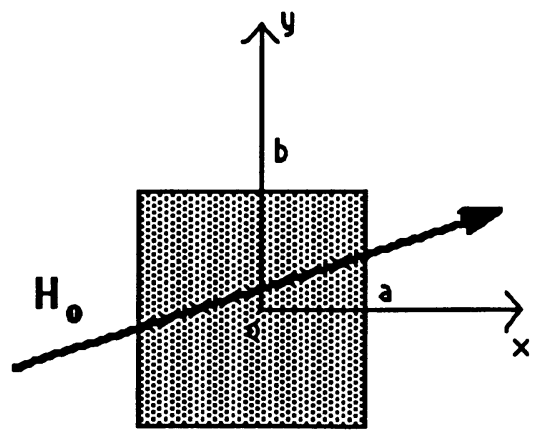

$\mathrm{f}=50 \mathrm{~Hz}$

$\mu=\mu$

épaisseur de peau: $\delta$

$2 \mathrm{a}=2 \mathrm{~b}$

Champ appliqué:

Hox $=$ Hoy

Champ de réation:

composantes $\mathrm{Hx}$ etHy calculées à la côte $y=b$

Fig. 5. - Champ de réaction des courants de Foucault.

[Reaction field of Eddy currents.] 


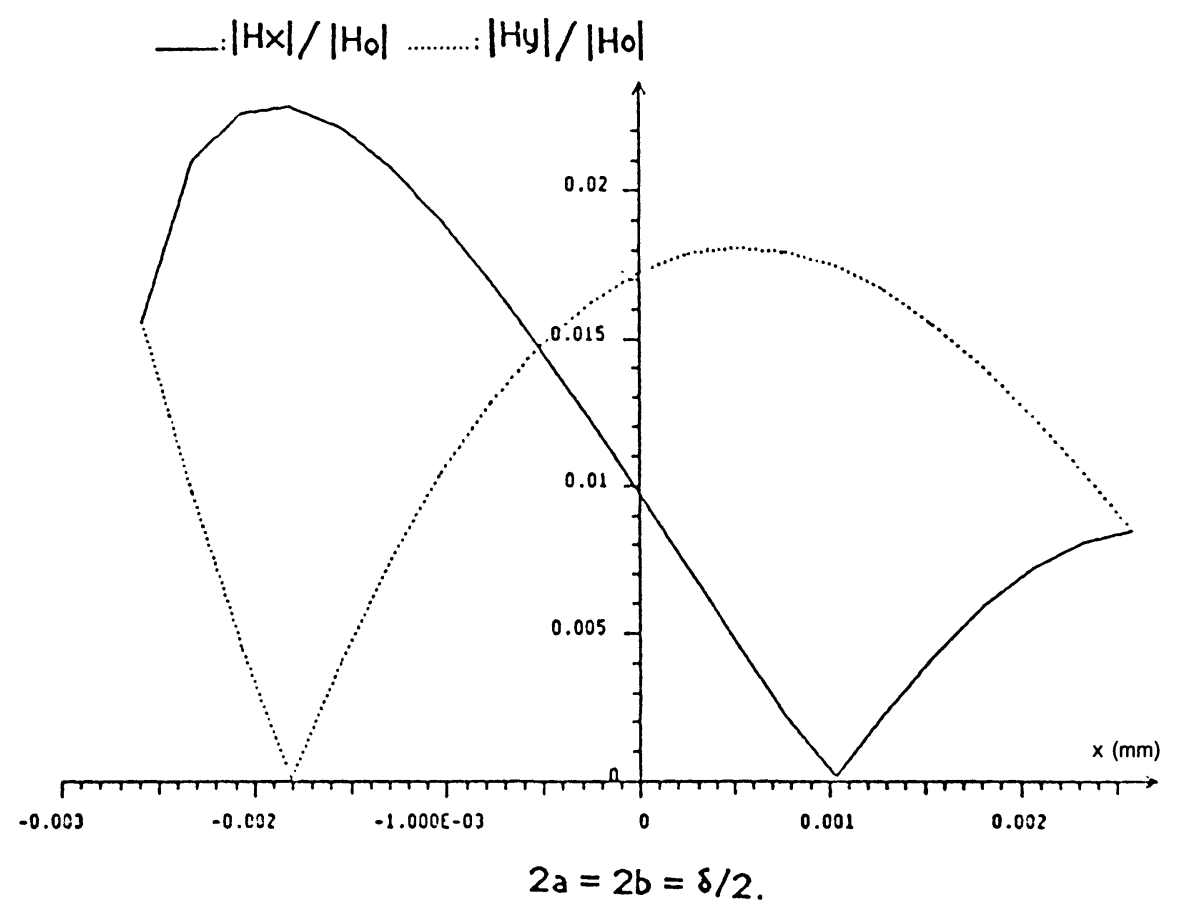

Fig. 5 (suite).

2.2.3 Aspect expérimental des pertes par courants de Foucault. - Dans le cadre de cette étude, une maquette de transformateur monophasé de 13,333 MVA a été construite.

Dans la pratique, les pertes supplémentaires sont mesurées de façon globale et il est difficile de les répartir entre les différentes sources de pertes supplémentaires. Dans notre démarche, nous avons essayé de limiter au mieux l'interférence de ces différentes sources. Pour ce faire, lors de la construction de la maquette, les parois de la cuve ont été placées suffisamment loin -des bobines et les parties proches des bobines ont été tapissées de shunts magnétiques surdimensionnés. Compte tenu de l'excellente qualité des tôles magnétiques, nous avons considéré que l'on peut raisonnablement négliger les pertes dans la cuve par rapport aux pertes dans les bobinages. Nous négligeons aussi les pertes dans le circuit magnétique parce que dans toutes les mesures que nous effectuons, les bobinages sont en court-circuit.

a maquette de transformateur qui nous a servi pour la mesure des pertes par courants de Foucault est constituée de quatorze bobines, dont six constituent la basse tension et sont reparties en deux groupes de bobines situées de part et d'autre des huit autres bobines qui constituent la haute tension (Fig. 3) ; ce type de bobinage où la basse tension est répartie de part et d'autre de la haute tension est qualifié de bobinage alterné.

Dans les bobines, la spire conductrice est constituée d'un brin conducteur unique. De la sorte, les pertes supplémentaires dans le bobinage se réduisent aux seules pertes par courants de Foucault. Pour connaître ces pertes, nous avons mesuré, d'une part, les pertes totales dans le bobinage, d'autre part, les résistances des différentes bobines; de ces dernières mesures, nous avons déduit les pertes par effet Joule et par différence, nous avons calculé les pertes par courants de Foucault. La concordance entre les résultats de mesures et les résultats de calculs est excellente.

Tableau I.

\begin{tabular}{|c|c|c|c|}
\hline \multicolumn{4}{|c|}{ Pertes résistives et pertes par courants de Foucault } \\
(en $\mathrm{kW}, \mathrm{à} 50 \mathrm{~Hz}$ ) \\
\hline Pertes & calculées & mesurées & Ecart relatif (\%) \\
\hline résistives totales & 75,53 & 76,12 & 0,7 \\
\hline totales par Foucault & 29,61 & 29,85 & 0,8 \\
\hline
\end{tabular}

\subsection{ModÈle DE CALCUl DES PERTES PAR COU- RANTS DE CIRCULATION}

2.3.1 Calcul des tensions induites sur les brins conducteurs. - Dans le but de réduire les pertes par courants de Foucault, les conducteurs sont habituellement subdivisés en brins. Ces brins conducteurs sont ensuite brasés ensemble, au moins au niveau des prises d'entrée et des prises de sortie du courant dans un groupe de bobines. L'opération a pour effet de créer des boucles dans lesquelles le champ de fuite induit des courants appelés courants de circula- 
tion. Nous désignons donc par courants de circulation, ces courants induits dont le trajet se referme entre des brins conducteurs brasés en parallèle. Autant les courants de Foucault sont des courants locaux, autant les courants de circulation méritent le qualificatif de courants globaux [2]. On peut considérer que ces courants de circulation sont dûs aux différences de tensions induites sur les différents brins conducteurs brasés en parallèle. Pour leurs parts, ces différences de tensions induites sont dues au fait que, les boucles formées par deux brins conducteurs quelconques ne voient pas le même flux total ; la différence de flux est occasionnée par le flux de fuite du transformateur.

L'amplitude du courant de circulation entre deux brins conducteurs brasés en parallèle sera d'autant plus importante que le sera celle de la différence des tensions induites sur es brins. Le calcul des tensions induites sur les différents brins conducteurs s'avère ainsi être important pour la représentation des pertes par courants de circulation.

D'après la loi d'induction de Faraday, la tension induite dans la boucle formée par un brin conducteur est donnée par :

$$
e=\mathrm{d}\left(\int_{s} \mathbf{B} \cdot \mathrm{d} \mathbf{s}\right) / \mathrm{d} t
$$

En plus de la connaissance du champ de fuite dans les différentes fenêtres du transformateur, tel que nous l'avons déterminé au paragraphe 2.1, l'application de la formule (10) nécessiterait la connaissance du champ magnétique dans le noyau magnétique. On peut considérer que, compte tenu du flux principal vu par toutes les boucles, il n'est pas nécessaire de connaître la distribution du champ magnétique dans le noyau magnétique, puisque notre intérêt porte essentiellement sur les différences de tensions induites et que le terme de flux commun disparaît dans de telles différences. Néanmoins, le calcul numérique de l'intégrale de surface qu'implique la relation (10) pourrait être coûteux en temps de calcul et entaché d'erreurs numériques importantes. D'une part, nous avons envisagé de nous affranchir de la connaissance du champ magnétique dans le noyau magnétique, d'autre part nous avons envisagé de remplacer l'intégrale de surface de la relation (10) par une intégrale curviligne. Compte tenu de la relation (4), nous appliquons le théorème de Stokes à la relation (10); ce qui conduit en régime harmonique de pulsation $\omega$ à écrire,

$$
e=i \omega \int_{\text {brin }} \mathbf{A} \cdot \mathrm{d} \mathbf{l} .
$$

Nous posons :

$$
e=i \omega \Phi
$$

où $\Phi$ désigne le flux induit sur le brin conducteur même si éventuellement, ce brin conducteur ne forme pas une boucle fermée. Désormais, pour calculer la tension induite sur un brin conducteur, il suffit de le suivre à travers les différentes fenêtres du transformateur, ce que permet la description que nous avons donnée de ces différentes fenêtres.

2.3.2 Transpositions dans les bobines. - Pour réduire les pertes par courants de circulation, des transpositions sont habituellement réalisées dans les bobines. Le but de ces transpositions est de réduire l'amplitude des différences de tensions induites sur les brins conducteurs brasés en parallèle, et par là, de réduire l'amplitude des courants de circulation entre ces brins.

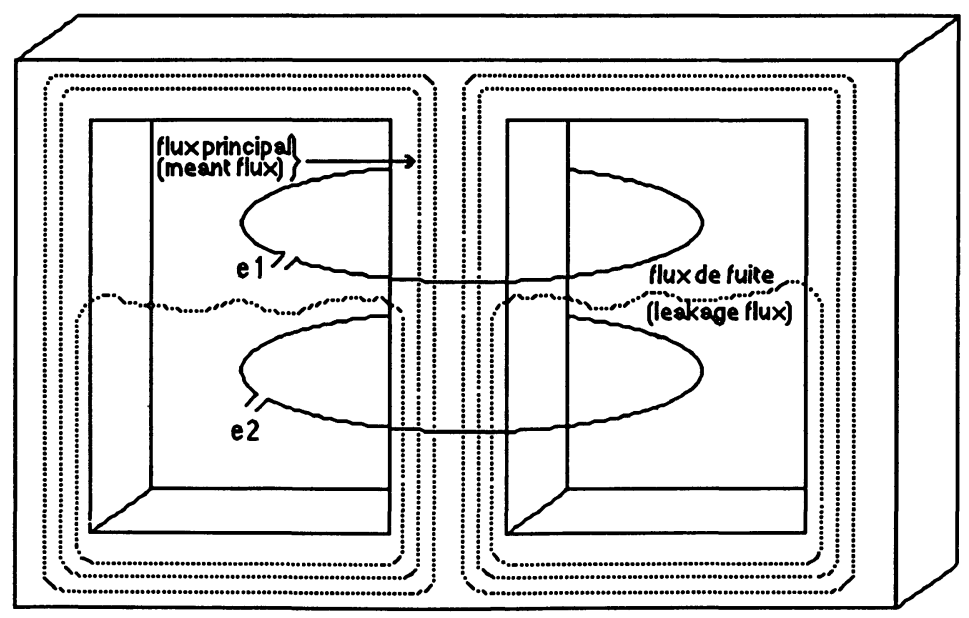

Fig. 6. - Influence du flux de fuite sur les tensions induites sur deux brins différents. A cause du flux de fuite, deux spires ne voient pas le même flux total.

[Effect of the leakage flux on the induced voltages of two strips. Due to the leakage flux, two turns do not see the same total flux.] 
Le principe des transpositions est simple à comprendre si l'on se réfère aux quelques illustrations que nous proposons ci-dessous.

Considérons par exemple une bobine dont la spire est constituée d'un certain nombre de couches de conducteurs brasés en parallèle. Les transpositions telles que nous les définissons, ont pour effet de permuter les brins d'une même couche de conducteurs, mais pas les brins de deux couches de conducteurs différentes. La figure 7 permet de visualiser l'effet d'une transposition sur une couche de conducteurs. Nous nous plaçons dans l'hypothèse où chaque couche de conducteurs est subdivisée en trois brins, et nous envisageons d'observer les positions des brins d'une couche avant et après la transposition en prenant le soin de numéroter ces brins.

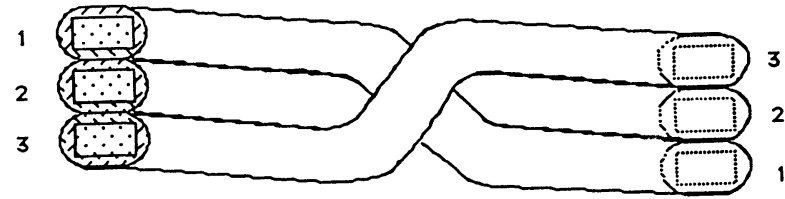

Fig. 7. - Transposition dans une bobine: vue d'une couche de conducteurs.

[Transposition in a coil : view of a layer of conductors.]

Nous proposons maintenant de justifier que l'on effectue des transpositions. Pour cela, considérons par exemple une bobine constituée d'une seule spire, subdivisée en deux brins conducteurs et plaçons-nous dans une configuration axisymétrique. Supposons que la valeur du potentiel vecteur soit $A_{1}$ sur la nervure centrale du brin numéro 1 et $A_{2}$ sur celle du brin numéro 2 . La longueur commune des deux brins étant $L$, on réalise une transposition à la distance $L_{1}$. Si nous négligeons l'épaisseur des brins, la tension induite vaut $A_{1} \cdot L_{1}+$ $A_{2} \cdot\left(L-L_{1}\right)$ sur le brin numéro 1 , et $A_{2} \cdot L_{1}+$ $A_{1}$. $\left(L-L_{1}\right)$ sur le brin numéro 2 . Dans cet exemple particulier, on constate que la différence des deux tensions induites est nulle lorsque la transposi-

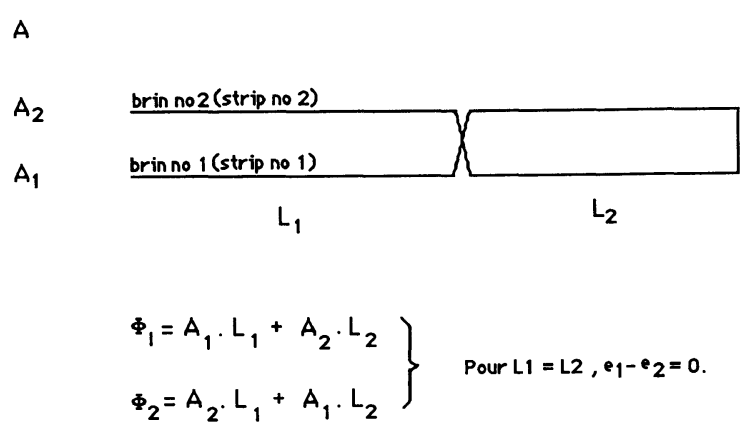

Fig. 8. - But des transpositions.

[Justifying transpositions.] tion est réalisée à une distance $L_{1}$ moitié de la longueur totale $L$.

Nous constatons alors que, lorsque les tensions induites sont calculées à partir de l'expression (11), la possibilité de suivre individuellement les brins conducteurs permet de prendre en compte les transpositions dans le calcul des tensions induites. Ce qui permet en retour de déterminer pour chaque bobine, l'emplacement de la transposition qui minimise les différences de tensions induites, ou les pertes par courants de circulation. Dans le cas d'un transformateur cuirassé, par rapport à l'exemple simpliste précédent, une difficulté supplémentaire est introduite dans la recherche des transpositions optimales, du fait que l'on est en présence de plusieurs fenêtres différentes.

2.3.3 Aspect expérimental des tensions induites sur les brins conducteurs. - Pour valider le modèle de calcul des tensions induites et le modèle de calcul des transpositions optimales, la maquette précédente est réutilisée et deux bobines supplémentaires d'épreuve sont insérées dans une région du transformateur où le champ de fuite est suffisamment intense et son orientation la plus favorable ; ceci correspond par exemple à un des deux espaces de passage entre un des groupes de bobines en basse tension et le groupe de bobines en haute tension (Fig. 3). Dans les deux bobines d'épreuve, le conducteur dans une spire est subdivisé en deux brins conducteurs court-circuités d'un côté; l'autre côté étant laissé ouvert pour mesurer la différence de tension induite. L'une des bobines est transposée à l'emplacement optimal calculé. L'autre n'est pas transposée et aucune des deux bobines d'épreuve n'est alimentée.

La tension calculée entre les deux brins de chaque bobine d'épreuve s'avère aussi en accord avec les résultats des mesures.

\section{Tableau II.}

Tensions induites entre les brins en volts, mesurées à $50 \mathrm{~Hz}$

Bobine Nombre Spire de Tension Tension Ecart relatif numéro de spires transposition calculée mesurée \%

$\begin{array}{rrrrrr}1 & 123 & 0 & 53,0 & 49,0 & 8,2 \\ 2 & 123 & 69 & 0,0 & 0,8 & \ldots\end{array}$

\subsubsection{Courants de circulation et pertes par courants} de circulation dans les bobines.

2.3.4.1 Cas de bobines indépendantes. - Pour une bobine dont tous les brins sont court-circuités en entrée et en sortie, on peut proposer le schéma électrique équivalent suivant :

Dans cette représentation qui est la plus générale, la tension induite sur un brin se calcule au moyen de 


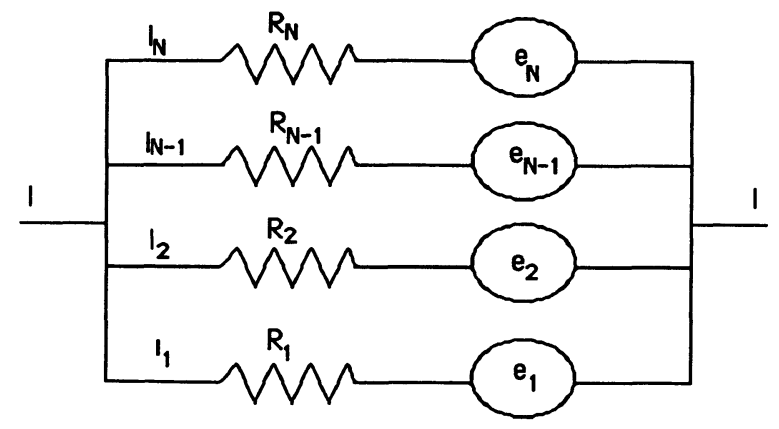

Fig. 9. - Schéma électrique équivalent d'une bobine court-circuitée à ses deux extrémités, avec: $N$, nombre total de brins dans la bobine $; I_{j}$, courant total dans le brin $\mathrm{n}^{\circ} j ; \boldsymbol{R}_{j}$, résistance en courant continu du brin $\mathrm{n}^{\circ} j$; $e_{j}$, tension induite sur le brin $\mathrm{n}^{\circ} j ; I$, courant total entrant dans la bobine.

[Equivalent electric diagram for a coil short-circuited at both ends, with : $N$, total number of strips in the coil ; $I_{j}$, total current in the $j$ th strip ; $R_{j}$, DC resistance of the $j$ th strip ; $I$, total current feeding the coil.]

l'équation (11). Pour ce calcul, nous avons utilisé un potentiel vecteur obtenu à partir du seul courant de charge dans les bobines comme nous le donne l'expression (9); ce qui signifie que nous avons négligé le champ de réaction dû aux courants de circulation. Il nous semble raisonnable de faire cette approximation, d'une part, à cause des transpositions qui réduisent considérablement l'amplitude des courants de circulation par rapport à ce qu'elle aurait été en l'absence des transpositions, d'autre part, et sans doute surtout, à cause de la proximité des brins d'une spire. En effet, la somme des courants de circulation dans les brins d'une spire étant nulle, le faible espacement de ces brins fait que les courants de circulation auront tendance à produire un champ de faible amplitude. Comme le montrent les courbes des figures 10, 11 et 12, des calculs faits a posteriori semblent justifier l'approximation qui vient d'être faite.

En écrivant les lois aux nœuds et les lois aux mailles du schéma électrique de la figure 9, le courant total dans le brin numéro $j$ est donné par l'expression :

$$
I_{j}=\frac{I}{R_{j} \sum_{k=1}^{N} \frac{1}{R_{k}}}+i \omega\left(\frac{\sum_{k=1}^{N} \frac{\phi_{k}}{R_{k}}}{R_{j} \sum_{k=1}^{N} \frac{1}{R_{k}}}-\frac{\phi_{j}}{R_{j}}\right)
$$

où les tensions induites ont été remplacées par leur expression d'après la relation (11). Le résultat de la sommation des premiers termes des courant $I_{j}$ sur tous les $N$ brins conducteurs d'une spire vaut I. Ces premiers termes représentent les courants qui circuleraient dans les brins s'il n'y avait pas de phénomène d'induction. La somme des seconds termes sur tous les $N$ brins conducteurs d'une spire est nulle. Ces seconds termes représentent les courants de circulation dans les brins conducteurs. En l'absence de pièces massives dans les fenêtres, il est aisé de constater en se basant par exemple sur la relation (7) et la relation (11) que, à $\pi$ près, les courants nominaux et le flux de fuite $\Phi$ sont en phase. Il apparaît alors d'après la relation (12) que les courants de circulation sont en quadrature avec le courant de charge $I$ et alors il est facile de montrer que les pertes résistives, les pertes par courants de Foucault et les pertes par courants de circulation peuvent être calculées séparément. En effet, nous avons montré plus haut que les pertes dues aux courants de Foucault peuvent être calculées séparément des pertes dues à tout courant dont la densité est uniforme sur la section d'un brin conducteur; la démonstration est alors terminée si l'on constate comme on vient de la faire que les courants de circulation sont en quadrature avec le courant de charge $I$.

$$
W_{\text {circulation }}=1 / 2 . \Sigma_{\text {brins }} R_{j} \cdot \operatorname{ABS}\left(I_{\text {circulation } j}\right)^{2} .
$$

\subsubsection{Connexions ou mariage de bobines consécu-} tives. - Quand les bobines comportent plusieurs couches de conducteurs en parallèle, les transpositions réalisées à l'intérieur des bobines ne suffisent pas à annuler les pertes par courants de circulation. En effet les transpositions telles que nous les avons décrites ci-dessus peuvent minimiser les tensions induites entre des brins conducteurs d'une même couche de conducteurs, mais compte tenu du fait que ces transpositions laissent invariantes les couches de conducteurs, elles n'ont presque pas d'effets sur les tensions induites entre les brins de deux couches de conducteurs différentes. En pratique, pour brasser au mieux le flux entre les différents brins conducteurs, on réalise des bobinages en câble continûment permuté, mais l'usage de ce type de câble peut être onéreux. Une solution intermédiaire consiste à réaliser des connexions particulières entre des paquets de brins de bobines consécutives ; ce qui permet aussi un brassage du flux induit entre les brins conducteurs. Les industriels qualifient cette opération de mariage des bobines. Plusieurs types de mariages peuvent être pratiqués mais les plus usités sont ceux dits de type groupe 6 et ceux dits de type groupe 4. En général des mariages ne sont réalisés qu'entre des bobines ayant le même nombre de couches de conducteurs par spire et ayant le même nombre de conducteurs par couche de conducteurs. Le nombre total de conducteurs dans une spire est en général multiple de six pour des mariages de type groupe 6 et multiple de 4 pour des mariages de type groupe 4.

La description qui a été donnée du transformateur permet d'identifier et de suivre chaque brin conduc- 
teur du bobinage; ce qui permet de donner une traduction logicielle aux mariages et cela étant, le schéma de calcul de pertes par courants de circulation précédent, où les bobines étaient indépendantes les unes des autres, se généralise aisément aux cas où des mariages sont réalisés entre les bobines.

2.3.4.3 Aspect expérimental des pertes par courants de circulation.

2.3.4.3.1 Cas d'un bobinage sans transposition et sans mariage entre les bobines. - Pour valider le modèle de calcul des pertes par courants de circulation et vérifier les influences des permutations et des mariages, nous reconsidérons la maquette qui a été utilisée dans les essais précédents. Cependant, cette fois-ci toutes les bobines sont remplacées par des bobines dans lesquelles la spire est constituée d'une seule couche de conducteurs subdivisée en six brins. Dans un premier temps, toutes les bobines sont court-circuitées chacune à ses deux extrémités ; ce qui signifie qu'il n'y a pas de mariages entre les différentes bobines. De plus, aucune transposition n'est réalisée dans les bobines. D'une part, les pertes totales dans les bobines ont été mesurées avec un wattmètre, d'autre part, les pertes par effet Joule dans les bobines ont aussi été mesurées, en mesurant les résistances des bobines. Nous avons ainsi obtenu par différence, la somme des pertes par courants de Foucault et des pertes par courants de circulation. En considérant que le calcul des pertes par courants de Foucault obtenu par l'expression (9) est désormais valide, compte tenu de la bonne concordance entre les résultats de calculs et les résultats de mesures obtenue au paragraphe 2.2 , nous avons déduit les pertes par courants de circulation par une seconde différence.

Tableau III. - Influence du champ de réaction dû aux courants de circulation.

\section{Pertes par circulation sans transposition et sans mariage des bobines (en $\mathrm{kW}$ et mesurées à $50 \mathrm{~Hz}$ )}

Spire de Pertes totales Pertes totales Ecart relatif permutation calculées mesurées en \%

La différence entre les résultats de calculs et les résultats de mesures atteint $20 \%$. Outre d'éventuelles erreurs de mesure, nous pensons qu'en l'absence de transpositions dans les bobines, et en l'absence de mariages entre les bobines, le rapport entre les courants de circulation et les courants de charge peut être important. C'est ce que nous avons vérifié par le calcul des courants de circulation dans les différents brins du bobinage.
Le champ de réaction dû aux courants de circulation se rouve alors ne pas être négligeable devant le champ de fuite dû aux courants de charge. Nous avons aussi essayé de vérifier cette assertion par le calcul ; et c'est ici qu'est apparu l'intérêt de pouvoir calculer le champ de fuite dans une fenêtre du transformateur, à partir des courants individuels des brins. Pour évaluer le champ de réaction des courants de circulation, il nous a alors suffi de réappliquer la même procédure de calcul, en remplaçant dans chaque brin, le courant de charge par le courant de circulation que nous avons déterminé dans ce brin d'après l'expression (12) et dans la suite, lorsque l'on réalise des mariages entre les bobines nous avons remplacé le courant de charge de chaque brin par son courant de circulation obtenu d'après les expressions équivalentes à (12). Pour la maquette de transformateur que nous avons décrit ci-dessus, nous avons trouvé que le rapport entre le champ de réaction des courants de circulation et le champ dû au courant de charge atteint des valeurs de l'ordre de $10 \%$ en certains points (Fig. 10).

On pourrait envisager de prendre en compte le champ de réaction dû auc courants de dirculation en procédant par exemple en plusieurs itérations successives dans le calcul du champ de fuite dans chaque fenêtre. Le principe de la méthode pourrait alors consister à partir du courant de charge dans les brins, de calculer le champ de fuite dans les différentes fenêtres par la procédure qui prend en compte le courant individuel des brins, de calculer ensuite les courants de circulation dus à ce champ de fuite et de corriger si cela se justifie, le courant dans les différents brins. Nous pensons qu'au bout d'un certain nombre d'itérations, en passant d'une itération à la suivante, la valeur du courant de circulation dans les différents brins ne devrait plus varier de façon notable ; ce qui revient à dire qu'au bout d'un certain nombre d'itérations le champ de fuite ne devrait plus varier de façon notable, d'une itération à l'autre. En exprimant ces idées de façon chiffrée, on peut établir un critère de convergence du processus. Nous n'avons pas essayé de mettre en œuvre ce processus itératif. En général, des transpositions et des mariages sont réalisés dans les bobines, ce qui a pour effet de réduire l'amplitude relative des cou-

l'amplitude du champ de réaction dû aux courants de circulation.

Les courbes de la figure 10 sont tracées dans la fenêtre numéro 1 à la côte $y=250 \mathrm{~mm}$; il en sera de même dans les figures 11,12 et 13 . La raison est que pour la maquette étudiée, la fenêtre numéro 1 présente un axe de symétrie par rapport à l'axe $y=250 \mathrm{~mm}$. Cet axe se trouve alors être un axe portant les valeurs maximales du champ de fuite dans la fenêtre numéro 1 ; c'est pour cette raison que nous l'avons choisi pour évaluer des champs de 


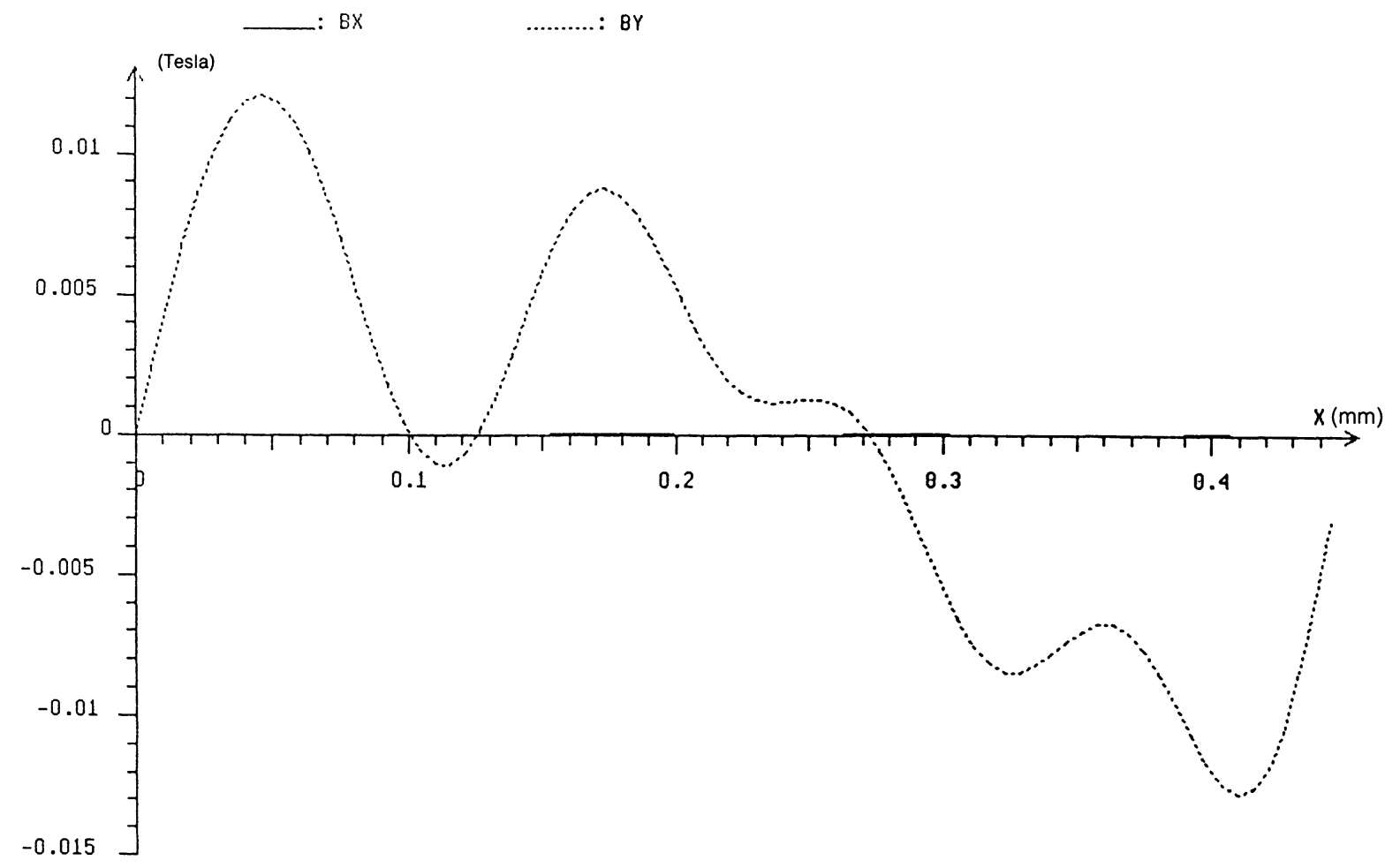

Fig. 10. - Champ de réaction dû aux courants de circulation, pour un bobinage sans transposition et sans mariage entre les bobines.

[Reaction field of circulating currents when there is no transposition in the coils and no interconnexion between the strips of consecutive coils.]

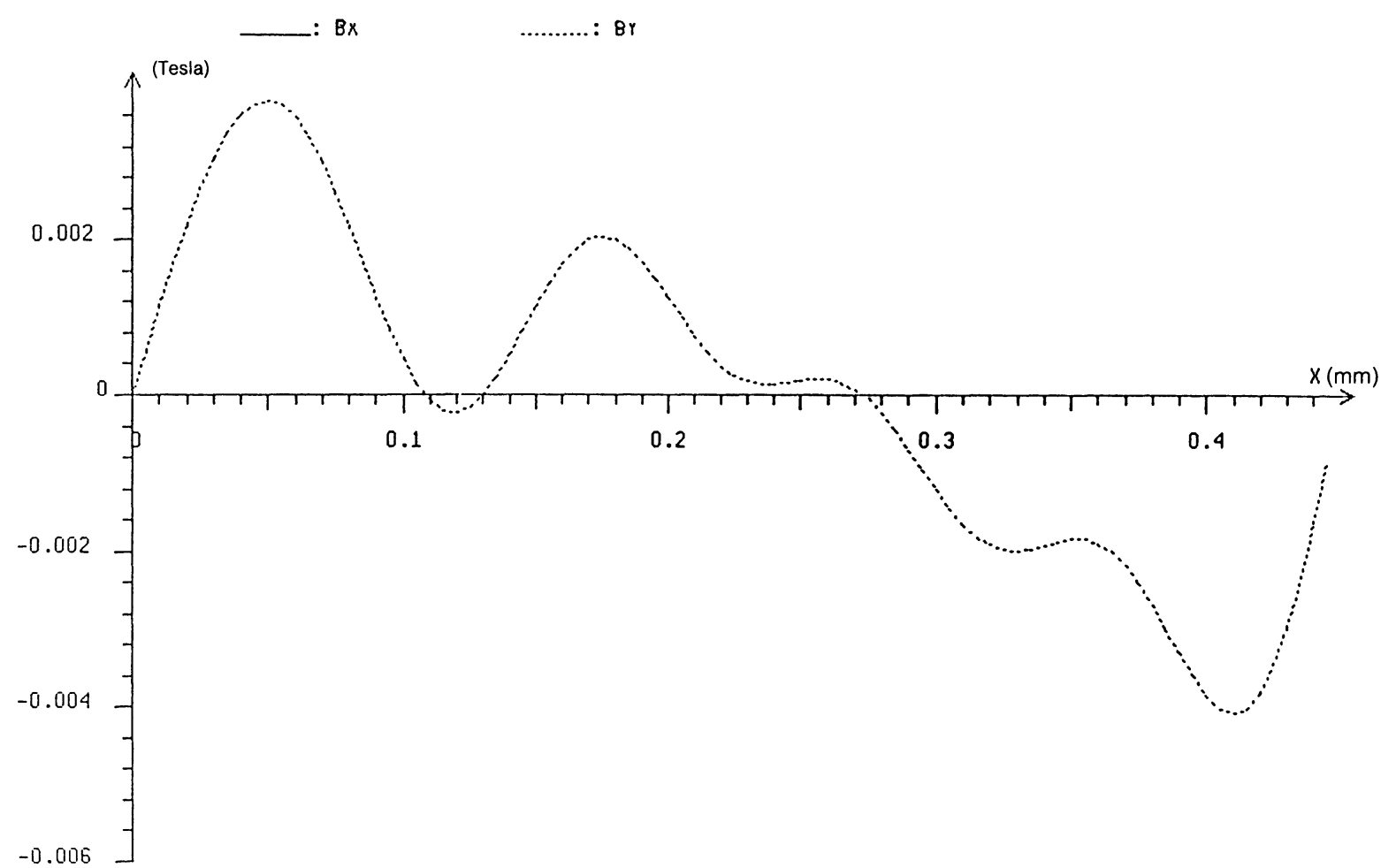

Fig. 11. - Champ de réaction dû aux courants de circulation, pour un bobinage sans transposition mais avec mariage des bobines en groupe 6 .

[Reaction field of circulating currents when there is no transposition in the coils and the strips of consecutive coils are interconnected (following a pattern called the «groupe 6 » pattern).] 


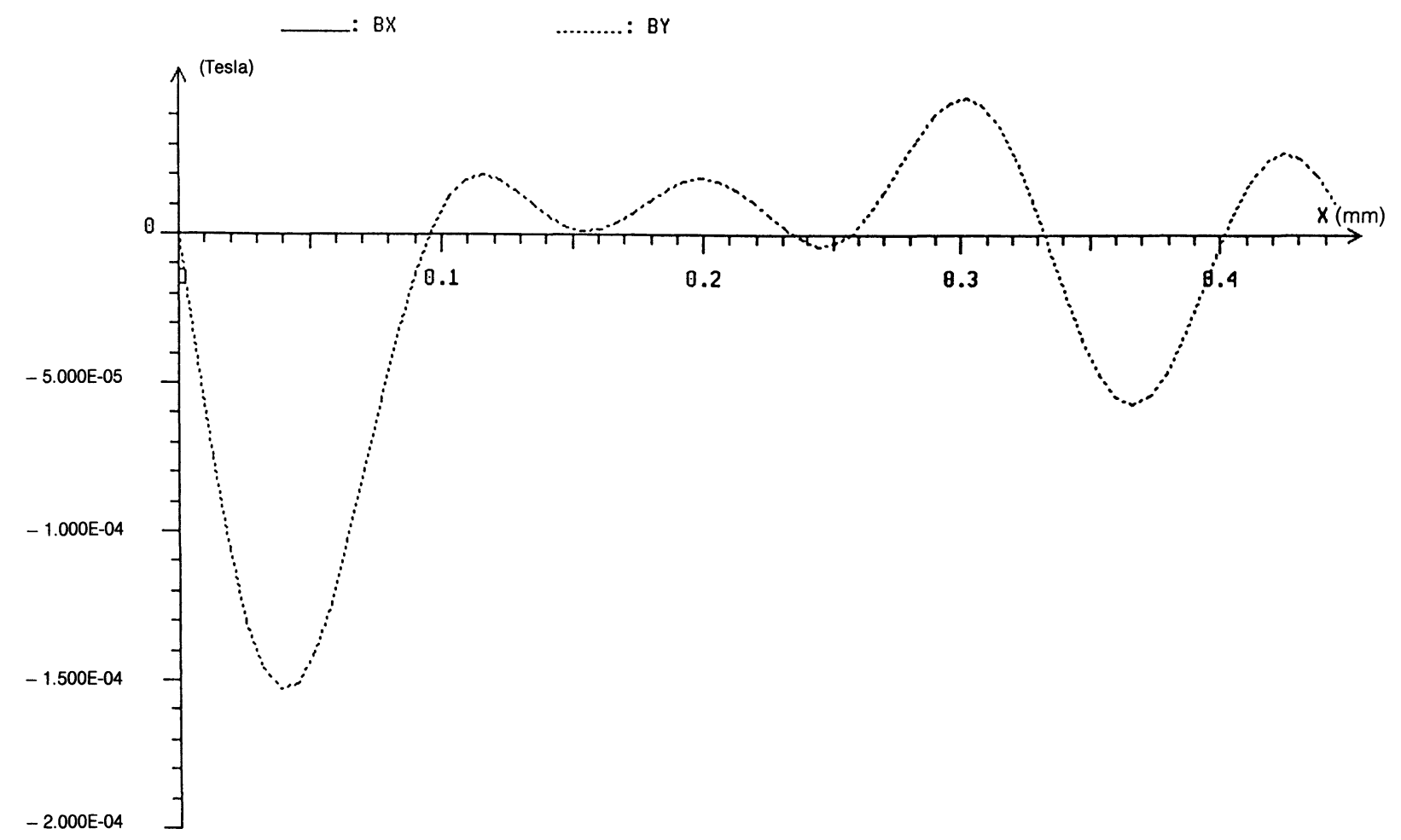

Fig. 12. - Champ de réaction dû aux courants de circulation, pour un bobinage avec transposition des bobines dans les spires optimales calculées et avec mariage des bobines (en groupe 6).

[Reaction field of circulating currents when the coils are transposed at the calculated right locations and the strips of consecutive coils are interconnected (following a pattern called the «groupe 6 » pattern).]

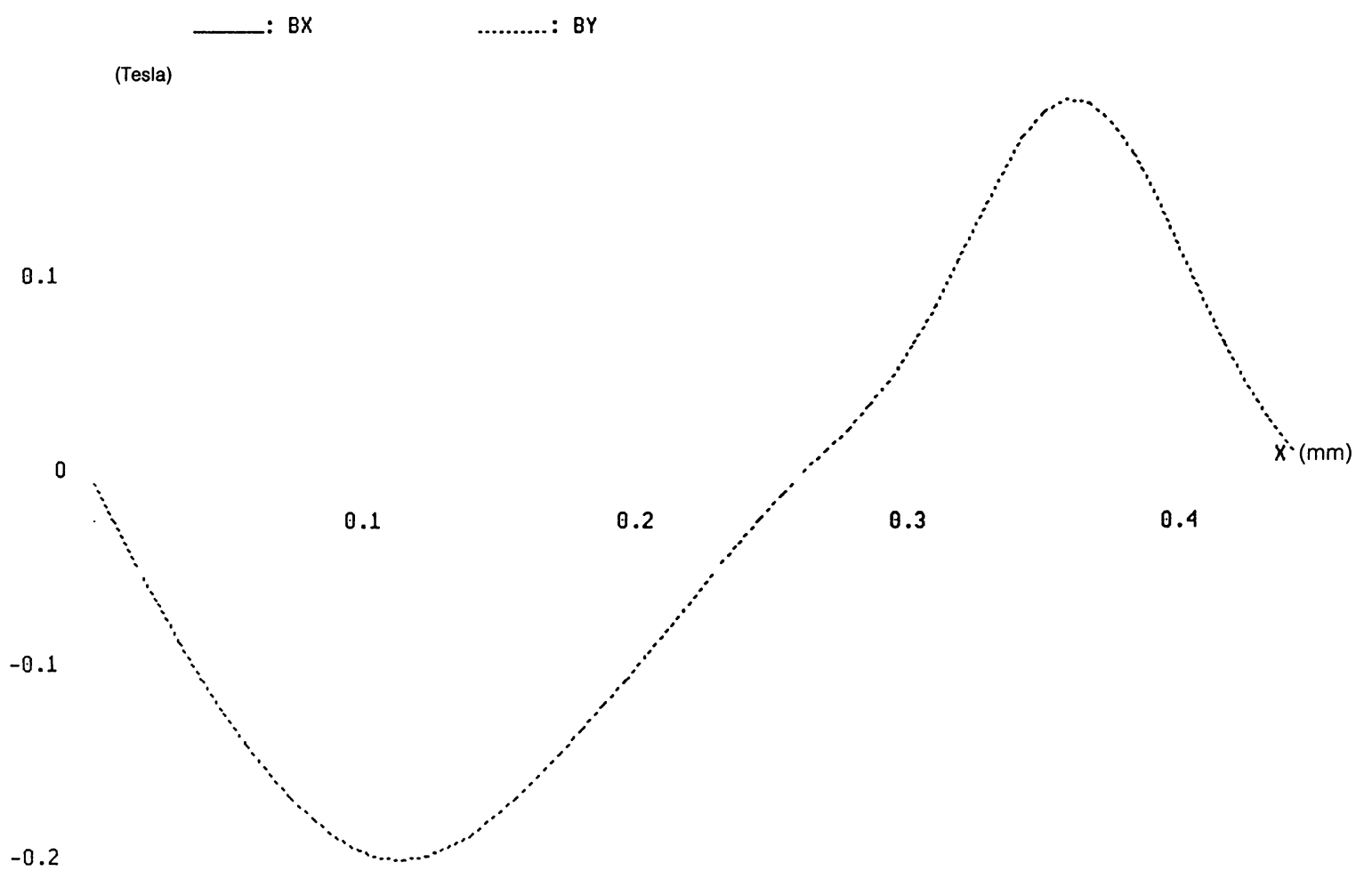

Fig. 13. - Champ de fuite dû aux courants de charge (champ de référence).

[Leakage field, due to the load currents (reference field).] 
réaction des courants de circulation. C'est aussi pour les mêmes raisons de symétrie que la composante $B_{x}$ du champ est nulle sur l'ensemble des figures 10 , 11,12 et 13 dont les courbes ont été tracées dans les mêmes conditions. Nous donnons sur la figure 13, des courbes représentant le champ de fuite dû aux courants de charge, pour permettre une meilleure appréciation des champs de réaction des courants de circulation.

2.3.4.3.2 Cas d'un bobinage sans transposition mais avec des mariages de type groupe 6 entre les bobines. - Un autre essai est réalisé sur la même maquette de transformateur que nous avons décrite dans les paragraphes précédents. Néanmoins, cette fois, des mariages de type groupe 6 , sont réalisés entre les bobines sans qu'aucune bobine ne soit transposée. Les résultats des mesures obtenus de la même manière que précédemment, concordent beaucoup plus avec les résultats des calculs que dans l'essai précédent.

Tableau IV. - Influence du champ de réaction dû aux courants de circulation.

Pertes par circulation pour bobinage sans transposition avec mariage en groupe 6

(en $\mathrm{kW}$ et mesurées à $50 \mathrm{~Hz}$ )

\begin{tabular}{|c|c|c|c|}
\hline $\begin{array}{c}\text { Spire de } \\
\text { permutation }\end{array}$ & $\begin{array}{c}\text { Pertes totales } \\
\text { calculées }\end{array}$ & $\begin{array}{c}\text { Pertes totales } \\
\text { mesurées }\end{array}$ & $\begin{array}{c}\text { Ecart relatif } \\
\text { en \% }\end{array}$ \\
\hline 0 & 18,95 & 18,3 & 3,5 \\
\hline
\end{tabular}

Nous avons vérifié par le calcul que le champ de réaction des courants de circulation est beaucoup moins important que dans l'essai précédent où on ne réalisait ni de mariage entre les bobines, ni de transposition dans les bobines; ce qui pourrait justifier la meilleure précision des résultats.

Dans un dernier essai, toutes les bobines sont transposées aux emplacements originaux calculés. Le faible ordre de grandeur des pertes par courants de circulation dans ce cas de figure a accru les risques d'imprécision et a rendu les mesures délicates. Nous avons cependant observé un fait intéressant que nous avons aussi obtenu par le calcul ; les valeurs des pertes par courants de circulation sont presque identiques; que les bobines soient mariées ou qu'elles soient chacune court-circuitée à ses deux extrémités. Cela tend à signifier que les bobines ont été transposées aux bons emplacements.

Tableau V. - Influence du champ de réaction dû aux courants de circulation.

\begin{tabular}{|c|c|c|c|}
\hline \multicolumn{5}{|c|}{$\begin{array}{c}\text { Pertes par circulation pour bobinage avec transpositions } \\
\text { et avec ou sans mariage } \\
\text { (en W et mesurées à } 50 \mathrm{~Hz})\end{array}$} \\
\hline $\begin{array}{c}\text { Spire de } \\
\text { permutation }\end{array}$ & $\begin{array}{c}\text { Pertes totales } \\
\text { calculées }\end{array}$ & $\begin{array}{c}\text { Pertes totales } \\
\text { mesurées }\end{array}$ & $\begin{array}{c}\text { Ecart relatif } \\
\text { en \% }\end{array}$ \\
\hline Optimale & 106 & 308 & 65 \\
\hline
\end{tabular}

Bien que la précision des résultats soit médiocre, nous avons obtenu par le calcul que le champ de réaction dû aux courants de circulation est bien négligeable devant le champ de fuite inducteur.

\section{Conclusion.}

Nous venons de présenter quelques méthodes de calcul des pertes supplémentaires dans les bobinages des transformateurs de puissance. Ces méthodes ont été éprouvées sur plusieurs versions d'un transformateur monophasé de 13,333 MVA construit pour les besoins de l'étude. Par ailleurs, ces méthodes ont été testées sur quelques transformateurs commerciaux. Globalement, nous avons obtenu d'excellents résultats.

Pour ce qui est des pertes par courants de circulation, les modèles que nous avons proposés ont semblé d'autant mieux représenter les phénomènes physiques que le nombre de couches de conducteurs par spire n'était pas trop élevé. Lorsque le nombre de couches de conducteurs par spire est devenu important, le calcul des pertes par courants de circulation est devenu moins précis en comparaison aux résultats de mesure disponibles. Il nous semble que cela est dû au fait que, d'une certaine façon, on est sorti du cadre de l'approximation bidimensionnelle. Par exemple, à cause des parties arrondies des bobines, la différence des longueurs des brins des différentes couches de conducteurs d'une même spire, peut devenir importante - contrairement à ce que suppose l'approximation bidimensionnelle. Dans ces cas, nous avons obtenu des résultats de calculs plus vraisemblables par rapport aux résultats des mesures, en uniformisant les longueurs des brins d'une même spire. 


\section{Bibliographie}

[1] Ngnegueu Tr., Meunier G., Sabonnadière J. C., Mailhot M., Arthaud J. P., Calculation of extra losses in power transformers windings, IEEE. Intermag conference (Vancouver, Canada) juillet 1988.

[2] Gueraud A., Contribution à l'étude des machines électriques à conducteurs dans l'entrefer, pp. 944. Thèse de doctorat, Université de Paris VI, juin 1988.

[3] STAFL M., Electrodynamics of electrical machines, Academia, Prague (1967).

[4] Binns K. J., LAWrenson P. J., Analysis and computation of electric and magnetic field, problems (Pergonen Press).

[5] Girgis R. S., Scott D. J., Heyne C. J., LANone T. B., Calculation of windings losses in shell form transformers ; for improved accuracy and reliability, IEEE Trans. Power delivery PWRD-2 (april 1987) $\mathrm{n}^{\circ} 2$.

[6] Thomas M. V., Mathematical model of load loss in shell form transformers, IEEE TPAS PAS 98 (Jan/Feb 1979) no 1.

[7] STOLL R. L., The analysis of eddy currents (Clarendon Press) 1974.

[8] Ngnegueu Tr., Contribution à la modélisation mathématique et informatique des pertes supplémentaires dans les transformateurs de puissance de type cuirassé, pp. 32-80, Thèse de doctorat, Institut National Polytechnique de Grenoble (Oct. 1989). 